\title{
Cancer-associated Fibroblasts and Modulation of the Antitumor Immune Response
}

\author{
Linda Ziani $^{1-3}$, Salem Chouaib ${ }^{1-3^{*}}$ and Jerome Thiery ${ }^{1-3}$ \\ ${ }^{1}$ INSERM, U1186, Villejuif, France \\ ${ }^{2}$ Gustave Roussy Cancer Campus, Villejuif, France \\ ${ }^{3}$ University Paris-Saclay, Faculty of Medicine, Le Kremlin Bicêtre, France \\ *Corresponding author: Salem Chouaib, Gustave Roussy Cancer Campus, Villejuif, France, Tel: 33142114211; E-mail: Salem.CHOUAIB@gustaveroussy.fr
}

Received date: December 12, 2015; Accepted date: December 20, 2015; Published date: December 27, 2015

Copyright: ( 2015 Ziani L, et al. This is an open-access article distributed under the terms of the Creative Commons Attribution License, which permits unrestricted use, distribution, and reproduction in any medium, provided the original author and source are credited.

\section{Description}

During tumor progression, tumor cells proliferate under adverse host conditions and use several survival strategies to block the action of key regulators of the immune response and circumvent anti-tumor defenses. Consequently, the current development of new immunotherapeutic strategies aimed at inducing or optimizing the immune response directed against the tumor and opened the way to new treatments of cancers. Besides the several known classical strategies used by tumor cells to escape to immune surveillance, it should be noted that the evasion of immunosurveillance by tumor cells is also under the control of the tumor microenvironment complexity and plasticity $[1,2]$.

Among the stromal cells, activated fibroblasts, termed cancerassociated fibroblasts (CAFs), play a critical role in the complex process of tumor-stroma interaction. CAFs, one of the prominent stromal cell population in most types of human carcinomas, are $\alpha$ SMA (alpha-smooth muscle actin) positive, spindle-shaped cells, who closely resemble normal myofibroblasts but express specific markers (ie, FAP (fibroblast-associated protein), PDGFR- $\beta$ (platelet-derived growth factor)) together with the fibroblastic marker FSP-1 (fibroblast specific protein 1) and vimentin (a mesenchymal marker). CAFs are also characterized by the absence of epithelial (cytokeratin, Ecadherin), endothelial (CD31) and fully differentiated smooth muscle (smoothelin) markers [3]. CAFs differentiate and proliferate in the tumor microenvironment in a transforming growth factor- $\beta$ (TGF- $\beta$ ), platelet-derived growth factor (PDGF) and fibroblast growth factor (FGF)-dependent manner from other cell types such as resident fibroblasts, mesenchymal stem cells, endothelial and epithelial cells $[4,5]$. In the tumor stroma, CAFs interact with tumor cells and secrete several factors such as extracellular matrix proteins (ie, collagen), matrix metallo-proteinases (MMPs), proteoglycans (ie, laminin, fibronectin), chemokines (ie, CXCL1, CXCL2, CXCL8, CXCL6, CXCL12/SDF1, CCL2 and CCL5), vascularisation promoting factors (ie, PDGF and VEGF) and other proteins which affect tumor cells proliferation, invasiveness, survival and stemness (ie, TGF- $\beta$, EGF, HGF, FGF, PGE2) [5]. Consequently, CAFs have been involved in tumor growth, angiogenesis, cancer stemness, extracellular matrix (ECM) remodelling, tissue invasion, metastasis and even chemoresistance [6,7].

During the past few years, these activated tumor-associated fibroblasts have also been involved in the modulation of the antitumor immune response by the secretion of immunosuppressive and pro-inflammatory factors (ie, TGF- $\beta$, IL-1 $\beta$, IL6, IL10...), chemokines (ie, CXCL12, CCL2...) and chemical mediators (ie, PGE2...) in the tumor microenvironment. As such, CAFs can potentially affect both innate and adaptive antitumor immune response [8,9]. For example, the secretion of CXCL12/SDF1 and CCL2/MCP-1 by CAFs is potentially involved in macrophages attraction in the tumor microenvironment and in their differentiation into a M2 immunosuppressive phenotype [10]. CAF secretion of chemokines can also recruit immunosuppressive myeloid-derived suppressive cells (MDSC) population to the tumor [11]. The secretion of TGF- $\beta$ by CAFs potentially affects dendritic cells biology by inhibiting their migration, maturation and antigen presentation capabilities, increases the numbers of regulatory $\mathrm{T}$ cells (Tregs) within the tumor microenvironment through the induction of FOXP3 expression [12] and interferes with cytotoxic $\mathrm{T}$ lymphocytes (CTL) function and frequency within the tumor [13]. The secretion of TGF- $\beta$ by CAFs can also attenuate IFN- $\gamma$ production by natural killer (NK) cells [14], as well as the expression of NK-activating receptors including NKG2D, NKp30 and NKp44 [12]. Similarly, the secretion of vascular endothelial growth factor (VEGF) by CAFs may affect dendritic function and increase the infiltration of Tregs and MDSC within the tumor [15]. Moreover, the secretion of prostaglandin E2 (PGE2) can decrease the expression of the activator receptor NKG2D on NK cells surface (which is also the case for indoleamine-2,3-dioxygenase (IDO) secretion by CAFs) [16] and induces FOXP3 expression in Tregs [17]. Nevertheless, further studies are clearly needed to fully elucidate the complex role of CAFs in the complex tumor immunosuppressive network.

Altogether, these findings highlight the action of CAFs on various levels of the antitumor immune response within the tumor microenvironment. Thus, combination therapy co-targeting CAFs and tumor cells or other immune check points (ie, PDL1, CTLA4) should represent a significant benefit in terms of tumor immunotherapy.

\section{Acknowledgement}

The authors are supported by the "Ligue Nationale contre le Cancer" (LNCC-Equipe Labélissée), the "Association pour la Recherche contre le Cancer" (ARC) and INSERM. L.Z. is supported by a PhD training fellowship from the "Ligue Nationale contre le Cancer" (LNCC).

\section{References}

1. Hasmim M, Messai Y, Ziani L, Thiery J, Bouhris JH, et al. (2015) Critical role of tumor microenvironment in shaping nk cell functions: implication of hypoxic stress. Front Immunol 6: 482.

2. Noman MZ, Hasmim M, Messai Y, Terry S, Kieda C, et al. (2015) Hypoxia: a key player in antitumor immune response. A Review in the Theme: Cellular Responses to Hypoxia. Am J Physiol Cell Physiol 309: C569-C579. 
Citation: Ziani L, Chouaib S, Thiery J (2015) Cancer-associated Fibroblasts and Modulation of the Antitumor Immune Response. J Mol Genet Med 9: 193. doi:10.4172/1747-0862.1000193

Page 2 of 2

3. Franco OE, Shaw AK, Strand DW, Hayward SW (2010) Cancer associated fibroblasts in cancer pathogenesis. Semin Cell Dev Biol 21: 33-39.

4. Augsten M (2014) Cancer-associated fibroblasts as another polarized cell type of the tumor microenvironment. Front Oncol 4: 62 .

5. Shimoda M, Mellody KT, Orimo A (2010) Carcinoma-associated fibroblasts are a rate-limiting determinant for tumour progression. Semin Cell Dev Biol 21: 19-25.

6. Valcz G, Sipos F, Tulassay Z, Molnar B, Yagi Y (2014) Importance of carcinoma-associated fibroblast-derived proteins in clinical oncology. J Clin Pathol 67: 1026-1031.

7. Ohlund D, Elyada E, Tuveson D (2014) Fibroblast heterogeneity in the cancer wound. J Exp Med 211: 1503-1523.

8. Harper J, Sainson RC (2014) Regulation of the anti-tumour immune response by cancer-associated fibroblasts. Semin Cancer Biol 25: 69-77.

9. Kraman M, Bambrough PJ, Arnold JN, Roberts EW, Magiera L, et al. (2010) Suppression of antitumor immunity by stromal cells expressing fibroblast activation protein-alpha. Science 330: 827-830.

10. Comito G, Giannoni E, Segura CP, Barcellos-de-Souza P, Raspollini MR et al. (2014) Cancer-associated fibroblasts and M2-polarized macrophages synergize during prostate carcinoma progression. Oncogene 33: $2423-2431$.

11. Tommelein J, Verset L, Boterberg T, Demetter P, Bracke M, et al. (2015) Cancer-associated fibroblasts connect metastasis-promoting communication in colorectal cancer. Front Oncol 5: 63.
12. Flavell RA, Sanjabi S, Wrzesinski SH, Licona-Limon P (2010) The polarization of immune cells in the tumour environment by TGFbeta. Nat Rev Immunol 10: 554-567.

13. Thomas DA, Massague J (2005) TGF-beta directly targets cytotoxic T cell functions during tumor evasion of immune surveillance. Cancer Cell 8: 369-380.

14. Laouar Y, Sutterwala FS, Gorelik L, Flavell RA (2005) Transforming growth factor-beta controls $\mathrm{T}$ helper type 1 cell development through regulation of natural killer cell interferon-gamma. Nat Immunol 6: 600-607.

15. Voron T, Marcheteau E, Pernot S, Colussi O, Tartour E, et al. (2014) Control of the immune response by pro-angiogenic factors. Front Oncol 4: 70 .

16. Balsamo M, Scordamaglia F, Pietra G, Manzini C, Cantoni C, et al. (2009) Melanoma-associated fibroblasts modulate NK cell phenotype and antitumor cytotoxicity. Proc Natl Acad Sci USA 106: 20847-20852.

17. Sharma S, Yang SC, Zhu L, Reckamp K, Gardner B, et al. (2005) Tumor cyclooxygenase-2/prostaglandin E2-dependent promotion of FOXP3 expression and CD4+ CD25+ T regulatory cell activities in lung cancer. Cancer Res 65: 5211-5220. 